\title{
Reabilitação estética em dente com descoloração escura da coroa ocasionada por traumatismo: relato de caso
}

Aesthetic rehabilitation of dark coronal discoloration following traumatic dental injuries: case report Rehabilitación estética de la decoloración coronal oscura después de lesione dental traumática: reporte de caso Valéria do Carmo de Brito LOPES Centro Universitário Católica de Quixadá (UniCatólica Quixadá), 63900-257 Quixadá - CE, Brasil https://orcid.org/0000-0002-4826-4123 Karlos Eduardo Rodrigues LIMA Centro Universitário Católica de Quixadá (UniCatólica Quixadá), 63900-257 Quixadá - CE, Brasil https://orcid.org/0000-0003-3127-9772

Talita Arrais Daniel MENDES Programa de Pós Graduação em Odontologia da Universidade Federal do Ceará, 60440-900 Fortaleza - CE, Brasil https://orcid.org/0000-0003-3519-3618

\section{Resumo}

Pacientes com alteração cromática dentária tendem a apresentar queixas e insatisfação significativa. Tendem a apresentar autoestima baixa, a restrição em sorrir, e a dificuldade de inserir-se na sociedade. Os incisivos superiores são os dentes que são impactantes no sorriso, sendo que estes possuem a característica de dominância, assim, qualquer alteração pode ser notada mais facilmente. Quando essas alterações ocorrem pode-se lançar mão de tratamento reabilitador estético com uso de resinas compostas. Logo, o presente estudo objetivou evidenciar a reabilitação estética e funcional através de um relato de caso clínico, a efetividade de facetas diretas e recontorno cosmético em um paciente com que apresente alteração cromática em dentes anteriores. Trata-se de um relato de caso clínico no qual foi realizado o atendimento a um paciente do gênero masculino, onde se diagnosticou escurecimento dentário por trauma, assim sendo, realizou-se um planejamento virtual do sorriso incluindo cirurgia gengival e restauração com resinas compostas. Desse modo, obteve-se um enceramento diagnóstico a partir do planejamento e em seguida realizou-se uma gengivectomia dos dentes anteriores superiores. Após o período de cicatrização realizou-se a faceta dentária do dente 11, seguido de fechamento de diastema entre os dentes 11 e 21 com recontorno cosmético. Por fim, pode-se perceber o sucesso do tratamento no reestabelecimento do sorriso do paciente, bem como melhora na autoestima do mesmo.

Descritores: Reabilitação Bucal; Pigmentação; Facetas Dentárias.

\section{Abstract}

Patients with dental chromatic changes tend to have complaints and significant dissatisfaction. They tend to have low selfesteem, the restriction on smiling, and the difficulty of being inserted in society. The upper incisors are the teeth that are impacting on the smile, and these have the characteristic of dominance, thus, any grounding can be noticed more easily. When these changes occur, aesthetic rehabilitation treatment using composite resins can be used. Therefore, the present study aimed to show aesthetic and functional rehabilitation through a clinical case report, the effectiveness of direct facets and cosmetic contouring in a patient with a chromatic alteration in anterior teeth. This is a clinical case report in which a male patient was seen, where traumatic dental darkening was diagnosed, thus, a virtual smile planning including gingival surgery and restoration with composite resins was carried out. Thus, a diagnostic waxing was obtained from the planning and then a gingivectomy of the upper anterior teeth was performed. After the healing period, the dental facet of tooth 11 was performed, followed by closing of the diastema between teeth 11 and 21 with cosmetic contour. Finally, the success of the treatment in reestablishing the patient's serissa can be seen, as well as an improvement in the patient's self-esteem.

Descriptors: Mouth Rehabilitation; Pigmentation; Dental Veneers.

\section{Resumen}

Los pacientes con cambios cromáticos dentales tienden a presentar quejas e insatisfacción significativa. Suelen tener baja autoestima, restricción para sonreír y dificultad para insertarse en la sociedad. Los incisivos superiores son los dientes que están impactando en la sonrisa, y estos tienen la característica de dominancia, por lo que cualquier conexión a tierra se puede notar más fácilmente. Cuando se producen estos cambios, se puede utilizar un tratamiento de rehabilitación estética con resinas compuestas. Por tanto, el presente estudio tuvo como objetivo mostrar la rehabilitación estética y funcional a través de un reporte de caso clínico, la efectividad de las facetas directas y del contorno cosmético en un paciente con alteración cromática en dientes anteriores. Se trata de un caso clínico en el que se atendió a un paciente masculino, donde se diagnosticó oscurecimiento dental por traumatismo, por lo que se realizó una planificación virtual de la sonrisa que incluyó cirugía gingival y restauración con resinas compuestas. Así, se obtuvo un encerado diagnóstico de la planificación y luego se realizó una gingivectomía de los dientes anteriores superiores. Después del período de cicatrización, se realizó la faceta dental del diente 11, seguida del cierre del diastema entre los dientes 11 y 21 con contorneado cosmético. Finalmente, es posible percibir el éxito del tratamiento en la restauración del seriso del paciente, así como una mejora en la autoestima del paciente.

Descriptores: Rehabilitación Bucal; Pigmentación; Coronas con Frente Estético.

INTRODUÇ̃̃O

O anseio por uma estética dental mais apurada trouxe uma busca desenfreada dos pacientes por um padrão estético préestabelecido, no qual vem propiciando o desenvolvimento de novos materiais e técnicas odontológicas mais conservadoras que visam procedimentos com resultados cada vez mais previsíveis e eficientes esteticamente ${ }^{1}$. A saúde bucal é relacionada com o bem estar do paciente e existe diversas formas de corrigir problemas relacionados a posição, alinhamento, simetria, textura e dentre os elementos que compõem a estética do sorriso, a cor dos dentes é de grande relevância ${ }^{2}$.

A alteração cromática, especialmente em incisivos superiores que sofreram trauma coronal gera grande desconforto e é percebido rapidamente, prejudicando a harmonia do sorriso, logo afetando o bem-estar e a autoestima $^{3}$. O escurecimento dental de um ou mais dentes, pode ter inúmeros fatores causais, como hemorragias pulpares, reabsorção dentária interna, administração de medicamentos como tetraciclinas, deposição de dentina secundária e terciária, redução da 
espessura de esmalte, má formação dental, traumatismo, entre outros ${ }^{4}$.

Devido a isso, a reabilitação estética de dentes escurecidos é um grande desafio, sendo muitas vezes ser necessário um planejamento multidisciplinar do tratamento que será executado, onde diferentes propostas de tratamento são oferecidas aos pacientes, nesses casos, alguns procedimentos reparadores são sugeridos desde os mais conservadores por meio de clareamento dentário, restaurações diretas com resina composta e/ou até mesmo os mais invasivos com utilização de facetas indiretas ${ }^{5}$.

A aplicabilidade das restaurações indiretas com cerâmicas odontológicas tem sido alvo de grande evolução no âmbito científico. A resistência à fratura, estabilidade da cor, estética, menor acúmulo de placa e biocompatibilidade são características vantajosas provenientes da sua utilização. No entanto, a grande maioria dos casos tem-se a remoção de esmalte, por menor que seja, podendo levar a exposição de dentina além de, alguns estudos associarem as restaurações indiretas a incidência de patologias pulpares e extensa perda de tecido coronal ${ }^{6}$.

No entanto, as restaurações diretas de resina composta são capazes de mimetizar a cor, forma e textura dos dentes por meio de estratificação anatômica, melhorando assim, a estética, além de exigir menor ou sequer nenhuma, dependendo do caso, preparação dentária, menor período de tratamento e baixo custo quando comparado à cerâmica, sendo, portanto, uma escolha de tratamento eficaz para pacientes com escurecidos decorrentes a trauma?

Portanto, o objetivo desse trabalho é relatar um caso clínico multidisciplinar de reabilitação estética de dentes anteriores que possui alteração cromática, demonstrando a importância do planejamento para obter resultados mais satisfatórios.

CASO CLÍNICO

O presente estudo foi submetido ao Comitê de Ética em Pesquisa e foi aprovado pelo parecer de número 4.586.072. Paciente HRB, sexo masculino, 22 anos de idade, procurou o serviço de atendimento odontológico queixando-se da estética do seu sorriso. Realizou-se o exame físico extraoral no qual não foi observada nenhuma alteração (Figura 1). Durante ao exame clínico intraoral foram observados dentes com coroa clínica curta, escurecimento dentário coronal no elemento 11, no mesmo dente observou-se uma restauração em excesso que estava provocando uma inflamação gengival na região com profundidade de sondagem de $3 \mathrm{~mm} e$ sangramento à sondagem (Figura 2).

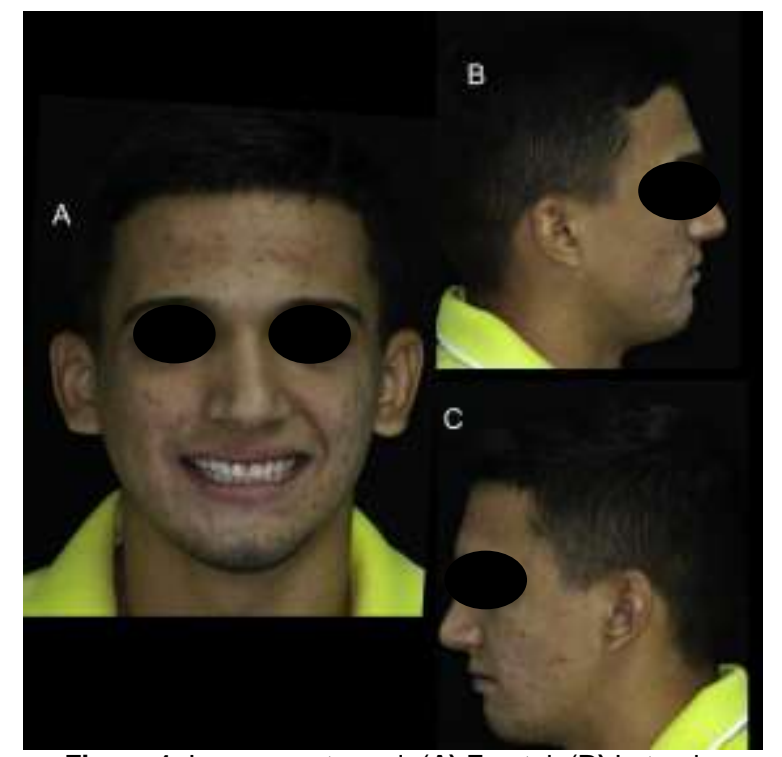

Figura 1: Imagem extraoral. (A) Frontal; (B) Laterais.

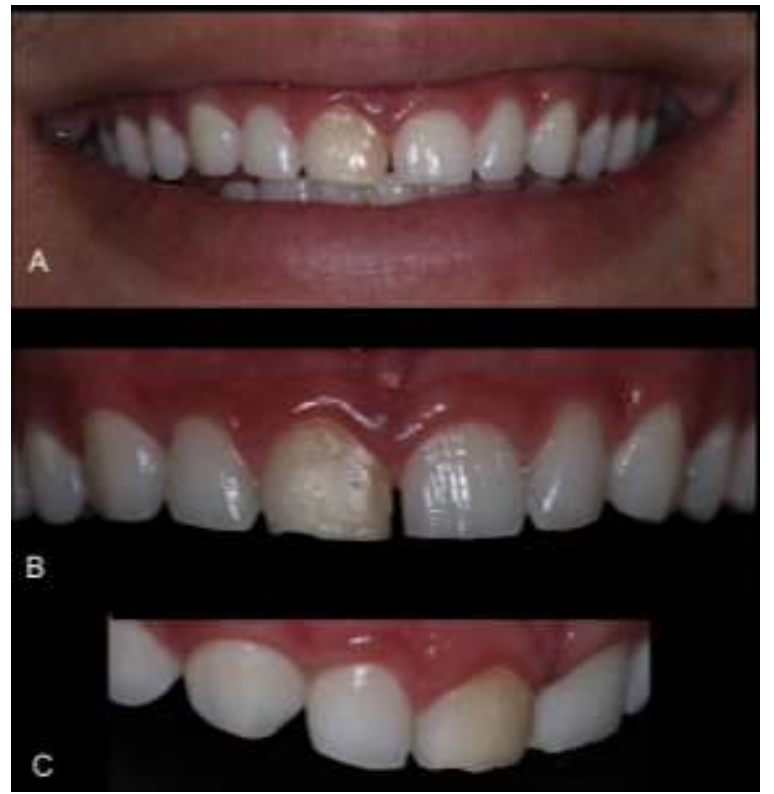

Figura 2: Aspecto frontal inicial do sorriso (A) e demonstração da inflamação gengival no elemento dentário 11 por excesso de resina (B e C).

O paciente também apresentava espaços interdentais, denominados de diastemas, além de desalinhamento de bordas incisais dos incisivos centrais superiores, e, também se constatou presença de cálculo supragengival em pequena quantidade na região de 33 à 43 . Chegou-se à um diagnóstico acerca do escurecimento dentário, pois 0 paciente referiu um trauma oriundo de acidente esportivo, diante disso, realizou-se um exame radiográfico intraoral do tipo periapical da região do dente 11 , sendo observada uma atresia no referido dente (Figura 3).

Mediante os exames clínicos realizados o plano de tratamento proposto consistiu em 
raspagem supra gengival para adequação a fim de remover fatores retentivos de placa, clareamento dental externo pela técnica de consultório com 0 uso de peróxido de carbamida à $35 \%$. Após isso, foi proposta a realização de um aumento de coroa clínica dos elementos dentário 14, 13, 12, 11, 21, 22, 23 e 24 , com o objetivo de melhorar a proporção largura e altura entre os dentes, confecção de faceta direta com uso de resina composta do elemento 11, seguido de reanatomizações estéticas e fechamento de diastemas com resina composta envolvendo os dentes 13, 12, 21, 22 e 23, baseando-se nos princípios de proporção áurea. Inicialmente, foi realizado o registro fotográfico completo necessário para fazer a análise e planejamento do caso.

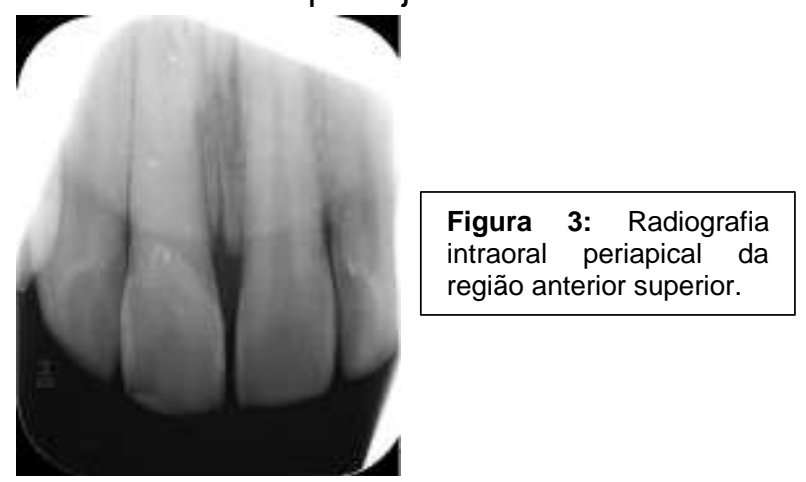

Após o plano de tratamento ter sido aceito pelo paciente foi realizada a moldagem das arcadas superior e inferior com alginato (HYDROGUM 5 COM 453G - ZHERMACK) para obtenção de moldes, sendo realizado o vazamento dos modelos com gesso pedra tipo III na proporção de $100 \mathrm{~g}$ para $30 \mathrm{ml}$ de água. Em seguida foi realizado um planejamento virtual do sorriso, seguindo os princípios de proporção estética e áurea, com o auxílio do software Power Point 2016 (Figura 4).

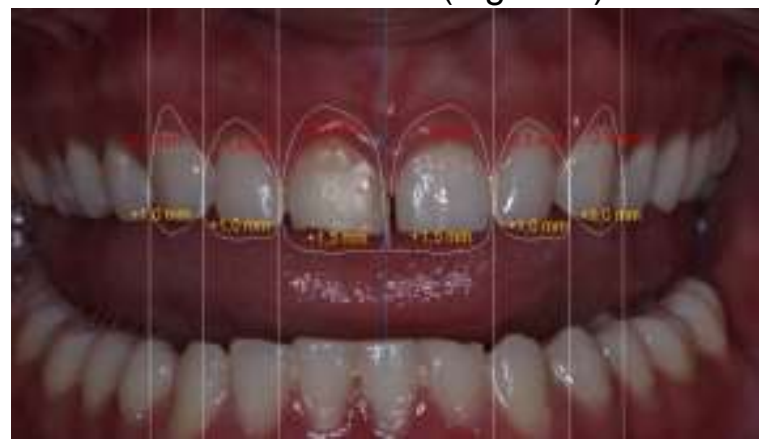

Figura 4: Planejamento virtual do sorriso do paciente.

A partir disso, efetuou-se então o enceramento diagnóstico dos dentes 14 a 24 (Figura 5) com o objetivo de confecção do guia para realização do processo restaurador, bem como para o procedimento cirúrgico do aumento de coroa clínica dos dentes anteriores superior. O enceramento diagnóstico foi realizado de acordo com o planejamento virtual do sorriso.

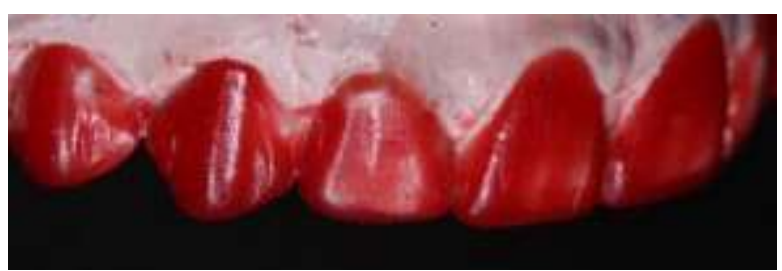

Figura 5: Enceramento diagnostico realizado com cera pegajosa, pela técnica progressiva.

Logo em seguida o paciente foi submetido aos exames periodontais de rotina a fim de acompanhamento. Após as seções de raspagem supragengival e orientações de higiene bucal foram obtidas condições de saúde periodontal, e, ao observar-se ausência de sangramento à sondagem, optou-se por iniciar o processo cirúrgico.

- Cirurgia periodontal de aumento de coroa clínica

Devido às características clínicas e anatômicas do contorno gengival, foi indicada a cirurgia de aumento de coroa clínica pela técnica de bisel interno na região anterossuperior, visto que o paciente se apresentava com saúde periodontal, profundidade de sondagem e nível de inserção adequados para o emprego de tal procedimento cirúrgico.

Para realização da cirurgia, inicialmente foi realizada a antissepsia intraoral utilizando bochecho de solução digluconato de clorexidina à $0,12 \%$ (Colgate, Jardim Jordão, Jaboatão dos Guararapes - PE, Brasil.) e extra oral com digluconato de clorexidina $2 \%$, para antissepsia (Colgate, Jardim Jordão, Jaboatão dos Guararapes - PE, Brasil.), seguido de anestesia terminal infiltrativa na região ântero-superior da maxila e isolamento do campo operatório com afastadores.

No ato cirúrgico foi realizada a demarcação da profundidade de sondagem da gengiva com uma sonda periodontal milimetrada Carolina do Norte (Figura 6A) e marcações dos pontos sangrantes da margem gengival na região mesial, distal e medial determinando a quantidade de gengiva a ser removida em direção a crista óssea. Em seguida foi realizada a incisão primária com auxílio de uma colher de molt (figura 6B), posteriormente foi realizada a incisão secundária com uso de lâmina de bisturí do tipo 15C (Swwan Morton, Sheffield England, Inglaterra) e cabo de bisturi número 05 , onde esta foi posicionada como bisel interno acompanhando as marcações em parábolas, dadas pela sonda periodontal, delimitando todo o tecido a ser removido e em seguida realizouse a incisão intra-sulcular. Após as incisões também foram confeccionadas com as mesmas lâminas, "colarinho" vestibular com medidas 
compatíveis com as medidas entre margem gengival (MG) e JCE citadas acima, que foi removido com curetas números 3 e 4 GoldmanFox (Trinity, São Paulo-SP, Brasil) (Figura 6C). A seguir, foi aplicado sobre a área cimento cirúrgico (Coe-PaK) e efetuado as orientações pós-cirúrgica, sendo a prescrição pós-operatória de analgésico: Dipirona $500 \mathrm{mg}$ de $6 / 6$ horas durante 24 (vinte e quatro horas), antiinflamatório não esteroidal: Ibuprofeno $600 \mathrm{mg}$ de $8 / 8$ horas durante 3 (três dias) e antisséptico bucal (Clorexedina 0.12\%), durante 7 (sete) dias, duas vezes ao dia, bem como cuidados locais de higiene. O pós-operatório de quinze dias mostrou aspecto clínico favorável, evidenciando boa cicatrização (Figura 6D).

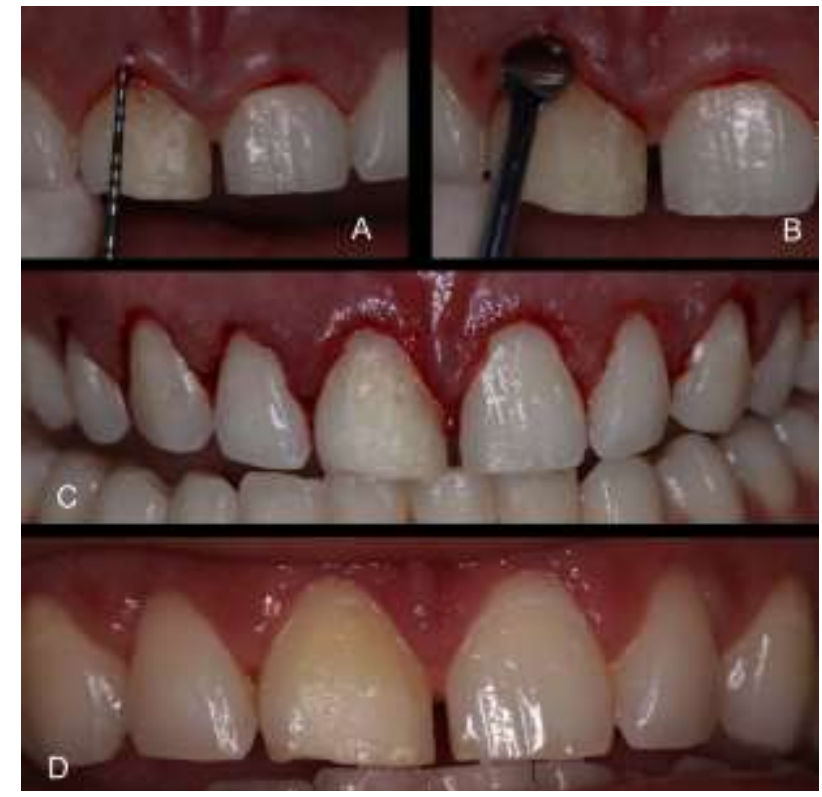

Figura 6: Sondagem da margem gengival com sonda milimetrada (A); Marcação dos pontos sangrantes e incisão primária com colher de Molt (B); Incisão em bisel interno e remoção do colarinho gengival (C); Cicatrização com 7 dias de cirurgia (D).

Após 30 (trinta) dias da cirurgia, 0 paciente voltou para consulta de reavaliação após cicatrização da gengivoplastia, tendo início o clareamento de consultório, em duas sessões com peróxido de hidrogênio 35\% (Whiteness HP Maxx, FGM), em três aplicações de 15 minutos. De início adaptou-se um afastador labial do tipo Expandex (Maquira), em boca, foi feito uma barreira gengival com Top Dam Blue (FGM), percorrendo o contorno gengival de todos os dentes, obtendo-se um campo de proteção. A barreira foi fotoativada por 20 segundos e logo em seguida foi efetuada a aplicação do dessensibilizante (Desensibilize KF 2\%) por 5 minutos na superfície dentária. Uma semana depois o paciente retornou para aplicação da segunda sessão de clareamento. Após a finalização do tratamento clareador, aguardouse um período de 15 dias para estabilização da cor e completa liberação de oxigênio residual ${ }^{8}$.
- Ajuste cosmético e procedimentos restauradores com resina composta

Iniciou-se 0 tratamento restaurador para reanatomização estética dos elementos dentários 12, 21 e 22 com recontorno cosmético e fechamento de diastema. Diante da conjunção de fatores (acentuada alteração de cor, forma e posição), foi indicada a realização de uma faceta direta com resina composta do elemento 11. Efetuou-se a profilaxia com pedra-pomes e água, utilizando escova de Robinson em baixa rotação. Posteriormente, foi realizado a seleção da cor por meio de um mapeamento cromático (Figura 7A). A resina composta escolhida foi a Empress Direct (Ivoclar Vivadent), na cor A1 para esmalte, A1 para dentina e Trans 20 para caracterização do terço incisal. Realizou-se, em seguida, 0 isolamento absoluto modificado (Figura 7B). Feito isso, inseriu-se subgengivalmente fios retratores de calibre 0.00 (Retractor) seguido de preparo para faceta por meio da confecção de canaleta na região cervical do dente com ponta diamantada esférica $n^{\circ}$ 1012. Em seguida, foram realizados os sulcos de orientação com broca troncocônica, sendo respeitados os planos de inclinação dos terços cervical, médio e incisal da face vestibular do dente. Por fim, foi efetuado o acabamento do preparo com pontas diamantadas de granulações finas e extrafinas $4138 \mathrm{~F}$ e 418FF (kG Sorensen) (Figura 7C), seguido do condicionamento com ácido fosfórico a 37\% (Condac 37, FGM, Joinville, SC, Brasil) nos dentes 12,11,21 e 22, respeitando o tempo de 30 segundos para o esmalte e 15 segundos para a dentina (Figura 7D).

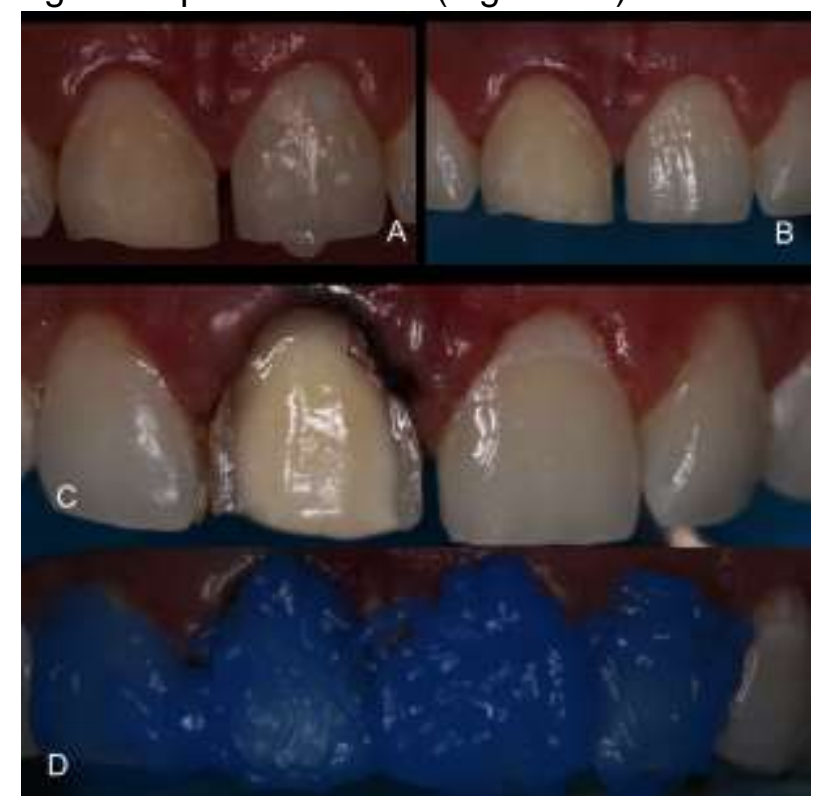

Figura 7: Aferição de cor por meio de mapa cromático (A); Isolamento do campo operatório (B); Preparo para faceta direta no dente $11(\mathrm{C})$; Condicionamento com ácido fosfórico a $37 \%$ dos dentes 11, 12, 21 e 22 (D).

$\mathrm{Na}$ sequência efetuou-se o controle da 
umidade com papel absorvente e o sistema adesivo (Adper Single Bond 2 - 3M) foi aplicado, conforme instruções do fabricante e, fotoativado por 20 segundos. Foi confeccionada uma guia com silicone de adição, obtida através do enceramento diagnóstico. Em seguida, efetuouse os incrementos de resina composta (Empress Direct-Invoclar), iniciando-se pela concha palatina com a resina trans 20 , seguida aplicou-se uma fina camada de opacificador no substrato escurecido (Figura 8). seguida de uma camada de dentina A1, posteriormente de esmalte A1 no elemento dentário de número 11. Realizou-se ainda o acabamento primário, texturização de anatomia e polimento com borrachas abrasivas e discos de feltro (Kit Diamond Master, FGM, SC, Brasil), objetivando um resultado final satisfatório (Figura 9 e 10).

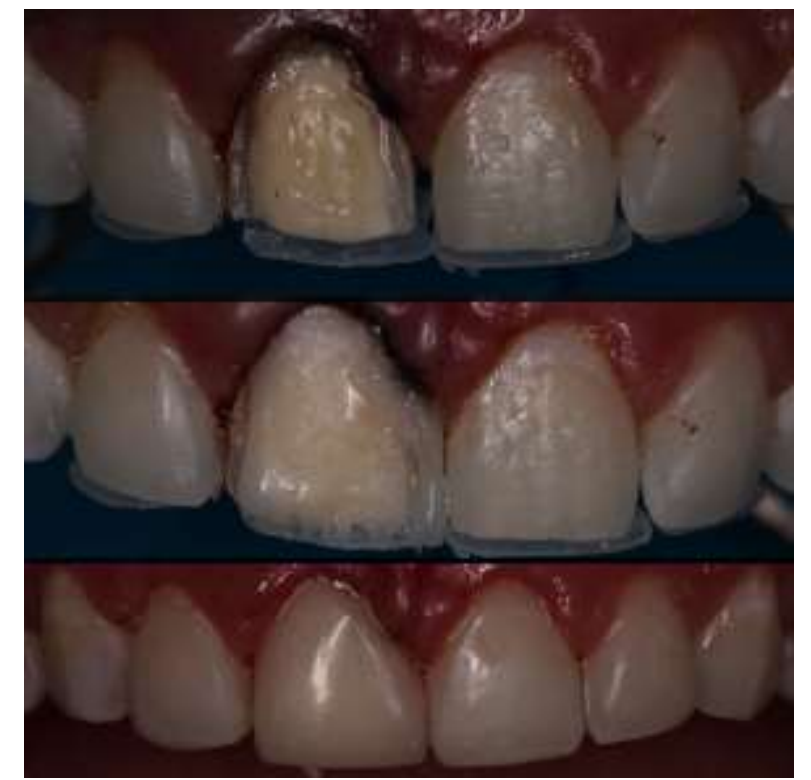

Figura 8: Realização da concha palatina com trans20, seguida da colocação do opacificador (A); Colocação de resina de dentina A1 (B); Colocação de resina A1 de esmalte (C).

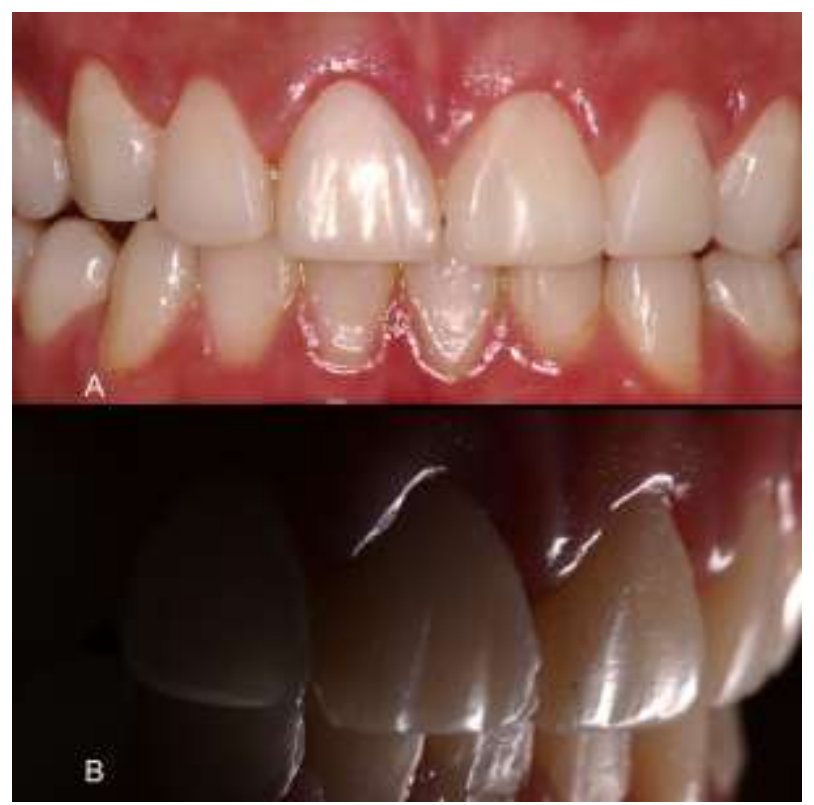

Figura 9: Aspecto final intraoral após acabamento e polimento $(A)$; Textura da superfície dentária (B).

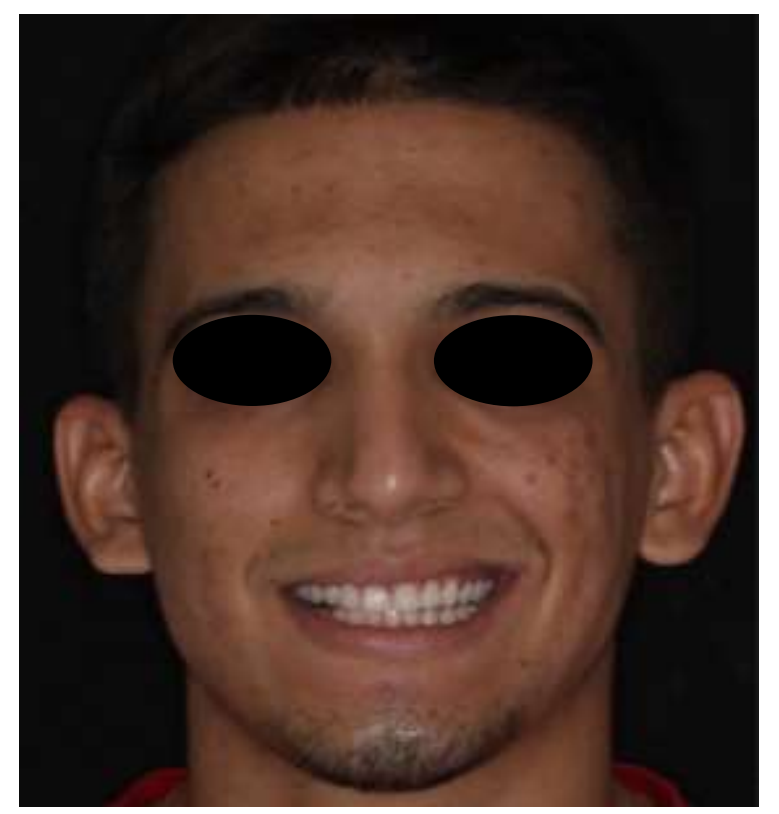

Figura 10: resultado final extraoral após 15 dias do processo restaurador.

DISCUSSÃO

A reabilitação oral envolvendo estética é um ramo bastante complexo, pois envolve conhecimentos de diversas áreas afins, como a dentística restauradora, endodontia, oclusão, materiais dentários, periodontia, bem como próteses. Esta reabilitação está intimamente relacionada com a harmonia entre as estruturas faciais, exigindo assim que o cirurgião-dentista esteja apto para estabelecer um bom diagnóstico frente à presença de alterações dentárias, sejam elas de origem cromática, forma, dentre outras, estabelecendo um tratamento adequado e consequentemente um prognóstico favorável para o paciente, já que é frequente a presença de alteração de cor, forma ou ambos, o que pode comprometer a estética e função ${ }^{9}$.

O diagnóstico dos diferentes tipos de alterações do esmalte e da dentina é possível a partir de uma anamnese detalhada e do conhecimento das características e fatores etiológicos de cada alteração. ${ }^{10}$ As alterações cromáticas em dentes anteriores é uma das principais causas de comprometimento estético, estas podem ser causadas por fatores sistêmicos, como hepatite neonatal, amelogênese e dentinogênese imperfeitas, hipoplasia de esmalte ou uso de tetraciclina durante gestação. Em relação aos fatores locais, são eles: necrose pulpar, presença de materiais obturadores, traumas ou agentes microbianos que induzem a deposição de dentina e, consequentemente, a calcificação pulpar $^{4}$.

No presente caso clínico, chegou-se ao diagnóstico de calcificação da cavidade pulpar 
relacionada ao traumatismo dentário oriundo, provavelmente de tratamento ortodôntico, que ocasionou o escurecimento coronal. Portanto, o prognóstico era favorável para confecção de faceta direta em resina composta e recontorno cosmético. A anamnese detalhada, bem como um exame físico acurado, fez com que se chegasse ao correto diagnóstico, minimizando os riscos de execução de um tratamento inadequado ${ }^{11}$.

Consolaro $^{12}$ relata que muitos ainda acreditam na tese que o tratamento ortodôntico possa causar necrose pulpar, calcificação, envelhecimento precoce e até mesmo o escurecimento dentário por obliteração pulpar, já que o rompimento dos feixes vasculares pode estar associado com o traumatismo dentário mediante a execução de movimentos dentários repentinos bruscos no tratamento ortodôntico e ao longo dos anos a polpa pode necrosar, levando ao escurecimento gradativo do dente traumatizado, sendo referida como metamorfose cálcica da polpa. Fachin et al. ${ }^{13}$ afirmaram que ocorre deposição de tecido mineralizado na periferia da câmara pulpar e no interior do tecido conjuntivo pulpar em dentes movimentados ortodonticamente, demorando certo tempo após o trauma para que essas calcificações sejam reconhecidas radiograficamente. $\mathrm{O}$ que se pode presumir que o presente caso trata-se de um escurecimento advindo de tratamento ortodôntico inadequado.

Como em qualquer procedimento odontológico em que se espera sucesso, o planejamento do caso é um passo que não deve ser menosprezado. Com o caso clínico em questão, observou-se que utilização do planejamento obtido através de um software DSD (Digital Smile Desing) torna o diagnóstico mais eficiente e possibilita um planejamento mais detalhado sobre a estética facial, dentogengival e dentária em cada etapa do tratamento reabilitador estético ${ }^{14}$. Contudo, torna-se de extrema importância que 0 cirurgião-dentista tenha conhecimentos das características e os princípios de proporções harmônicas da relação dento-facial para execução de um trabalho adequado ${ }^{15}$, como no presente relato de caso, no qual lançou-se mão da realização do procedimento de planejamento digital utilizando outro software, mas que desempenhou as mesmas funções de estabelecimento das relações harmônicas faciais e dentárias.

O planejamento virtual do sorriso torna o tratamento reabilitador mais previsível, tornando o processo clínico mais descomplicado para o cirurgião dentista, bem como o paciente fica ciente de como o tratamento vai se comportar. ${ }^{14}$ O sucesso clínico do tratamento proposto depende da integração harmônica entre tecidos moles e rígidos, sendo importante que o profissional considere a prevalência da proporção áurea e estética para obtenção de dimensão de um sorriso agradável ${ }^{15,16}$.

Neste caso clínico, optou-se por um planejamento de tratamento cirúrgico com gengivoplastia tanto pela saúde bucal do paciente como pela boa quantidade de gengiva, sendo uma técnica cirúrgica de fácil execução, realizando assim a remoção do excesso de tecido gengival com intuito de posicionar a margem gengival de forma harmônica com o lábio superior, proporcionando melhor simetria no que diz respeito a altura e largura dos dentes, e fazendo com que a estética ideal fosse alcançada. Silva et al. ${ }^{17}$ relatam que, além de objetivos estéticos, a cirurgia periodontal oferece remodelamento plástico do tecido gengival para repor de forma anatômica o contorno fisiológico adequado, visando facilitar a higiene bucal, bem como a obtenção de uma melhor estética.

Existe uma variedade de opções disponíveis para recuperação estética de dentes escurecidos, e a decisão por um material adequado sempre causa dúvidas ${ }^{18}$. A literatura refere como vantagens a confecção de facetas que podem ser realizadas em uma única sessão clínica, pois requer pouco ou até mesmo nenhum desgaste do dente, consequentemente, se tem melhor aceitação por parte do paciente, permite ao cirurgião-dentista estabelecer desde a seleção de cor até a morfologia final, além de que, é de baixo custo e apresentam maior conservação da estrutura dental comparado a restaurações indiretas, dispensando etapas laboratoriais, além de não requererem a confecção de um material provisório ${ }^{19}$. Deve-se salientar que o presente caso clínico lançou mão do uso de material restaurador direto ao paciente, por todas essas vantagens citadas anteriormente.

Porém, as facetas de resina composta direta possuem algumas desvantagens, incluindo a instabilidade de cor, acarretando escurecimento ao longo do tempo, desenvolvimento de sensibilidade devido a contração de polimerização, levando ao surgimento de fendas marginais e consequentemente, a microinfiltração, baixa resistência à abrasão e acúmulo de placa, porosidade, e dificuldade de mascarar o substrato escurecido ${ }^{20}$. Corroborando com o estudo de Ryan et al. $^{21}$, um dos fatores a ser considerado para a longevidade e sucesso 
clínico de dentes tratados com resina composta é a escolha do tipo de resina a ser utilizada. Existem no mercado várias marcas com diferentes propriedades, em que as características de opacidade, translucidez, resistência, brilho e polimento devem ser consideradas.

Nesse caso clínico, foi utilizado resinas da Empress Direct - Ivoclar, um compósito de restauração nano-híbrido que em sua composição possui um bom desempenho clínico, dentre suas características pode-se citar: menor contração de polimerização, propriedades mecânicas satisfatórias, comportamento óptico, melhor brilho, excelente lisura superficial e melhor estabilidade de cor, resultando em uma melhor estratificação, mimetizando precisamente o elemento dental do ponto de vista estético e funcional, pode-se ressaltar que o método de escolha foi o não VITA, lançando mão de um mapeamento cromático que pode gerar uma seleção de cor mais acurada ${ }^{22}$.

O desafio para 0 sucesso de restaurações estéticas em dentes que apresentam escurecimento é a busca pelo mascaramento. Quando a resina não é capaz de mascarar por completo uma lesão com alteração de cor, o uso de pigmentos opacificadores se faz necessário e é bastante eficaz $^{23}$. No presente caso clínico, em virtude do grau de escurecimento do substrato, foi necessária a utilização de um opacificador previamente à aplicação de resina composta para dentina. Ryan et al. $^{21}$ reforçam a necessidade de conhecimentos sobre opacidade e translucidez dos diferentes sistemas de resina composta, já que resinas ditas como opacas não apresentaram um grau de opacidade compátivel com a dentina humana que sofreram injurias, consequentemente sendo dessa forma justificável a utilização de opacificadores resinosos em dentes com alterações cromáticas acentuadas, tornando-se capaz de reproduzir com fidelidade características ópticas auxiliando o trabalho com resinas composta.

Grande número de procedimentos clínicos odontológicos é intermediado por sistemas adesivos, sendo ele um passo bastante importante para realização do tratamento restaurador adequado, uma vez que, a longevidade das restaurações pode estar interligada com a integridade da interface de união dentina e adesiva ${ }^{24,25}$. No entanto, desde o surgimento da técnica de condicionamento ácido, a adesão em esmalte é efetiva, apresentando durabilidade e sucesso clínico, ao contrário da adesão em dentina, que se apresenta menos inerente e presumível, por dispor de mais conteúdos orgânicos, além disso há degradação de fibras colágenas presentes na camada híbrida, tornando a união resinadentina um procedimento muito sensível à técnica adesiva e materiais que são utilizados. Logo, o profissional deve estar apto frente a escolha do sistema adesivo adequado cada caso clínico particular, entendendo o mecanismo de ação de cada um deles ${ }^{26}$.

Para que uma restauração com resina composta seja considerada ideal ela precisa reproduzir de forma satisfatória não só a função e estética como já citado, mas também conferir as características superficiais adequadas da estrutura dentária como cor e textura, proporcionando também uma lisura superficial próxima a do esmalte dentário. A rugosidade de superfice, oriunda de acabamento e polimento inadequados, pode resultar em menor estabilidade de cor, aumento de desgaste e acúmulo de placa ${ }^{27}$. Nesse contexto, o acabamento e polimento são definidos por serem processos que auxiliam na finalização do tratamento estético, proporcionando melhor vedamento marginal entre dente e restauração, além de contribuir na remoção de irregularidade, reduzindo o acúmulo de placa. Logo, remove a pigmentação extrínseca e a colonização microbiana, favorecendo assim brilho e lisura, aumentando a longevidade dessas restaurações ${ }^{27}$.

Portanto, o sucesso na obtenção de um resultado estético satisfatório não está associado apenas às propriedades do material e à habilidade técnica, mas deve incluir um correto planejamento, buscando melhorar os resultados clínicos em longo prazo para assim prover um melhor prognóstico ${ }^{18}$.

CONCLUSÃO

Pode-se constatar que é possível a realização de reconstrução adesivas diretas em dentes com alteração cromática e obtenção de resultados satisfatórios, desde que sejam utilizados bons materiais restauradores associados a uma correta indicação e execução da técnica, reestabelecendo assim a estética do sorriso, a funcionalidade dos dentes na arcada e a inclusão social do paciente.

Ao final do tratamento, observou-se harmonia do elemento 11 em relação aos demais dentes da região anterior da arcada, tanto do ponto de vista cromático, quanto anatômico. Dessa forma, em casos de associação de técnicas operatórias e restauradoras com resina composta a um bom planejamento resulta em uma reabilitação 
estética satisfatória, solidificando um caso duradouro e bem-sucedido.

REFERÊNCIAS

1. Haddad MF, Rocha EP, Assunção WG. Cementation of prosthetic restorations: from conventional cementation to dental bonding concept. J Craniofac Surg. 2011;22(3):952-58.

2. Gresnigt MMM, Kalk W, Ozcan M. Randomized controlled split-mouth clinical trial of direct laminate veneers with two micro-hybrid resin composites. J Dent. 2012;40(1):755-66.

3. Lucena MTL, Mantovani M, Fracalossi C, Silva GR. Internal bleaching in devitalized teeth with walking bleach technique - case report. UNINGÁ Review. 2015;24(1):33-9.

4. Fonseca AML. Clareamento dental com a utilização do laser: um estudo controlado de boca dividida. [Dissertação de Mestrado]. Rio de Janeiro: Universidade Estácio de Sá; 2011.

5. Hickel R, Peschke A, Tyas A, Mjor I, Bayne S, Peters $M$ et al. Clinical criteria for the evaluation of direct and indirect restorations. Update and clinical examples. J Adhes Dent. 2015;12(1): 259-68.

6. Simão Júnior BS, Barros CCP. Reabilitação estética com faceta indireta em porcelana. ROPLAC. 2012;2(1):9-15

7. Fernandes HGK, Silva R, Marinho MAS, Oliveira POS, Ribeiro JCR, Moyses MR. Evolução da resina composta: Revisão da Literatura. Revista da Universidade Vale do Rio Verde. 2014;12(2):401-11.

8. Brignall I, Mehta SB, Banerji S, Millar BJ. Aesthetic composite veneers for an adult patient with amelogenesis imperfecta: a case report. Dent Update. 2017;38(9):594-603.

9. Abduo J, Lyons K. Clinical considerations for increasing occlusal vertical dimension: a review. Aust Dent J. 2012;57(1):2-10.

10. Passos IAC, Costa JDMC, Melo JM, Forte FDS, Sampaio FC. Defeitos do esmalte: etiologia, características clínicas e diagnóstico diferencial. J Health Sci Inst.2007;25(5):187-92.

11. Cerri A, Guarim JA, Genovese WJ. Planning and diagnosis in Dentistry with the bioethical principles. Rev Assoc Paul Cir Dent. 2015; 69(3):217-25.

12. Consolaro A. Alterações pulpares induzidas pelo tratamento ortodôntico: dogmas e falta de informações. Dental Press Ortodon Ortop Facial. 2007;12(1):15-7.

13. Fachin EVF, Luisi SB, Borba MG. O processo de calcificação pulpar. Rev ABO Nac. 2001/2002;9(6):347-51.

14. Omar D, Duarte C. The application of parameters for comprehensive smile esthetics by digital smile design programs: A review of literature. Saudi Dent J. 2018;30(1):7-12.

15. Garcia $P$, Costa RG, Calgaro M, Ritter AV, Correr GM, Cunha LF et al. Design digital e técnica de mock-up para o planejamento de tratamentos estéticos com facetas laminadas de porcelana. J Conserv Dent. 2018;21(4): 455-58.

16. Araújo Jr EM, Fortkamp $S$, Baratieri LN. Closure of diastema and gingival recontouring using direct adhesive restorations: a case report. J Esthet Restor Dent. 2009;2(4):229-40.

17. Silva DB, Zaffalon GT, Corazza PFL, Bacci JE, Oliveira CS, Magalhães JCA. Cirurgia plástica periodontal para otimização da harmonia dentogengival - relato de caso clínico. Braz J Health. 2010;1(1):31-6.

18. Santo CC, Matafora FL, Bitencourt EL, Moro AFV. Harmonização do sorriso com lentes de contato: realto de caso clínico. Clin Int J Braz Dent. 2014;10(4):410-18.

19. Gonçalves R, Correia I, Ferreira JC, Pires P, Carvalho MT, Pina-Vaz I. Descoloração dentinária: aplicação de facetas componeer. Rev Port Estomatol Med Dent Cir Maxilofac. 2015;56(2):132-38.

20. Fahl N. Coronal Reconstruction of a severely compromissed central incisor with composite resins: a case report. J Cosmet Dent. 2010; 26(1):92-113.

21. Ryan EA, Tam LE, McComb D. Comparative translucency of esthetic composite resin restorative materials. J Can Dent Assoc. 2010: 76-84.

22. Mendonça DHS, Azevedo MLC, Leandrini JC, Souza-Gabriel AE. Functional aesthetic treatment of crown fracture in anterior teeth with severe crowding. RSBO. 2012;9(3):328-33.

23. Hoeppner MG, Pereira SK, Neto ES, Camargo LNG. Tratamento estético de dente com alteração cromática: faceta direta com resina composta. Publ UEPG Ci Biol Saúde. 2003; 9(3/4):67-72.

24. Aranha ACC, Mitsui FHO, Marchi GM. Facetas diretas em resina composta pós-microabrasão - Relato de caso clínico. J Bras Dent Estet. 2003;2(5):72-8.

25. Alothman Y, Bamasoud MS. The success of dental veneers according to preparation disgn and material type. Open Access Maced J Med Sci. 2018;6(12):2402-6.

26. Reis AF, Giannini M, Lovardino JR, Ambrosano GM. Effects of various finishing system on the surface roughness and staining susceptibility of packable composites resins. Dent Mater. 2003; 19(1):12-8.

27. Wolff D, Kraus T, Schach C, Pritsch M, Mente $\mathrm{J}$, Staehle HJ, et al. Recontouring teeth and closing diastemas with direct composite buildups: a clinical evaluation of survival and quality parameters.J Dent. 2010;38(12):1001-9. 
CONFLITO DE INTERESSES

Os autores declaram não haver conflitos de interesse

AUTOR PARA CORRESPONDÊNCIA

Talita Arrais Daniel Mendes

Rua Monsenhor Furtado, S/N, Rodolfo Teófilo,

60430-355 Fortaleza - CE, Brasil

E-mail: talita_arrais@hotmail.com

Submetido em 13/05/2021

Aceito em 18/07/2021 